\title{
In vivo antitumor activity by dual stromal and tumor-targeted oncolytic measles viruses
}

\author{
Yuqi Jing ${ }^{1} \cdot$ Valery Chavez $^{1} \cdot$ Natasha Khatwani $^{1,2} \cdot$ Yuguang Ban $^{3} \cdot$ Andrea P. Espejo $^{4} \cdot$ Xi Chen $^{3} \cdot$ Jaime R. Merchan $^{1}$
}

Received: 25 October 2019 / Revised: 27 February 2020 / Accepted: 10 March 2020 / Published online: 31 March 2020

(c) The Author(s), under exclusive licence to Springer Nature America, Inc. 2020

\begin{abstract}
The tumor stroma acts as a barrier that limits the efficacy of systemically administered oncolytic viruses (OV). We previously demonstrated that stromal-selective, retargeted oncolytic measles viruses (MVs) delay in vivo tumor progression. To further characterize the contribution of stromal targeting to MV's overall in vivo efficacy in an experimental cancer model, a dual targeted oncolytic measles virus (MV-CD46-muPA) able to simultaneously infect murine stromal (via murine uPAR) and human cancer (via CD46) cells was developed. MV-CD46-muPA infected, replicated, and induced cytotoxicity in both murine and human cancer cells. Viral infection was successfully transferred from stromal to tumor cells in vitro, leading to tumor cell oncolysis. Systemic administration of MV-CD46-muPA led to improved antitumor effects in colon (HT-29) cancer xenografts compared to vehicle or CD46 only targeted MVs. These effects were associated with improved tumor viral deposition, increased apoptosis, and decreases in murine stromal endothelial cells and fibroblasts. MV-CD46muPA modulated cell cycle, survival, proliferation, and metabolic pathways, as determined by functional proteomic analysis of treated tumors. The above findings further validate the concept that dual stromal and tumor cell viral targeting enhances the therapeutic effects of systemically administered OVs and support further preclinical and clinical development of stromal directed virotherapies.
\end{abstract}

\section{Introduction}

Oncolytic viruses (OVs) represent promising options for the treatment of advanced malignancies, with an increasing number of viral platforms undergoing late stage clinical trials [1], and one OV agent (Talimogene

Supplementary information The online version of this article (https:// doi.org/10.1038/s41417-020-0171-1) contains supplementary material, which is available to authorized users.

Jaime R. Merchan

jmerchan2@med.miami.edu

1 Division of Medical Oncology, University of Miami Miller School of Medicine and Sylvester Comprehensive Cancer Center, Miami, FL, USA

2 Sheila and David Fuente Graduate Program in Cancer Biology, University of Miami Miller School of Medicine, Miami, FL, USA

3 Division of Biostatistics and Bioinformatics, Sylvester Comprehensive Cancer, Center, University of Miami Miller School of Medicine, Miami, FL, USA

4 Division of Internal Medicine, University of Miami Miller School of Medicine, Miami, FL, USA
Laherparepvec) approved in the United States [2]. OVs offer a potential advantage over other forms of anticancer therapies, as they can selectively infect, replicate, and induce cytotoxicity in tumor cells, with subsequent induction of antitumor immunity [2-4]. The oncolytic measles virus (MV) platform is a promising one, having demonstrated safety and early evidence of promising clinical antitumor activity (including complete responses) in phase 1 clinical trials $[5,6]$. There are three known receptors used by MV to mediate cell entry, CD150 (SLAM), CD46, and more recently, the epithelial receptor nectin-4 [7]. However, there are several important challenges that limit the full potential of OVs in the clinic. They include suboptimal target specificity after systemic administration, virus clearance by host immune responses, and inadequate tumor entry and spread due to the tumor stroma [8-10]. Although significant advances in virus retargeting and modulation of antiviral immunity have been made [9,11-15], overcoming the tumor stroma to improve OV entry and spread remains a significant challenge. Significant effort has been devoted into overcoming the stromal barrier, including the design of viral vectors that target angiogenesis, or combination of $\mathrm{OVs}$ and 
a
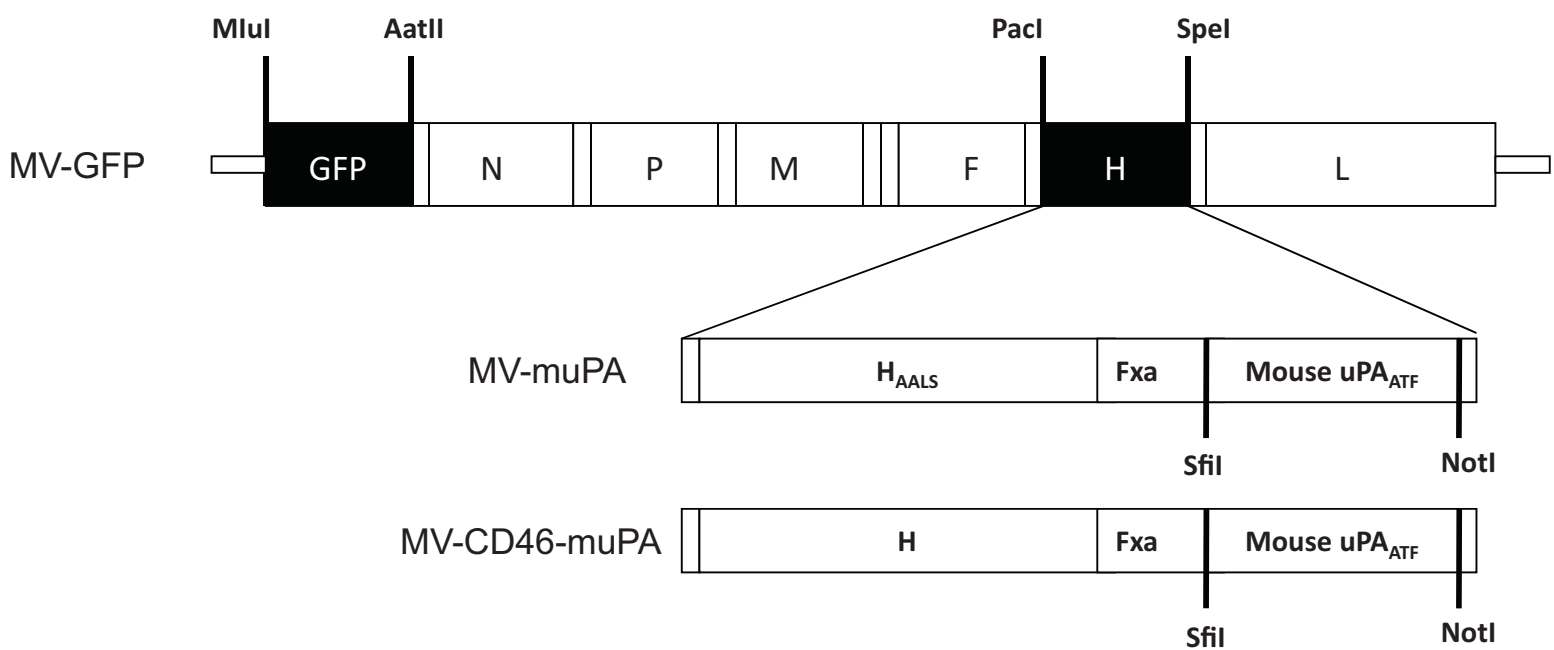

b MDA-MB-231 786-O HT-29
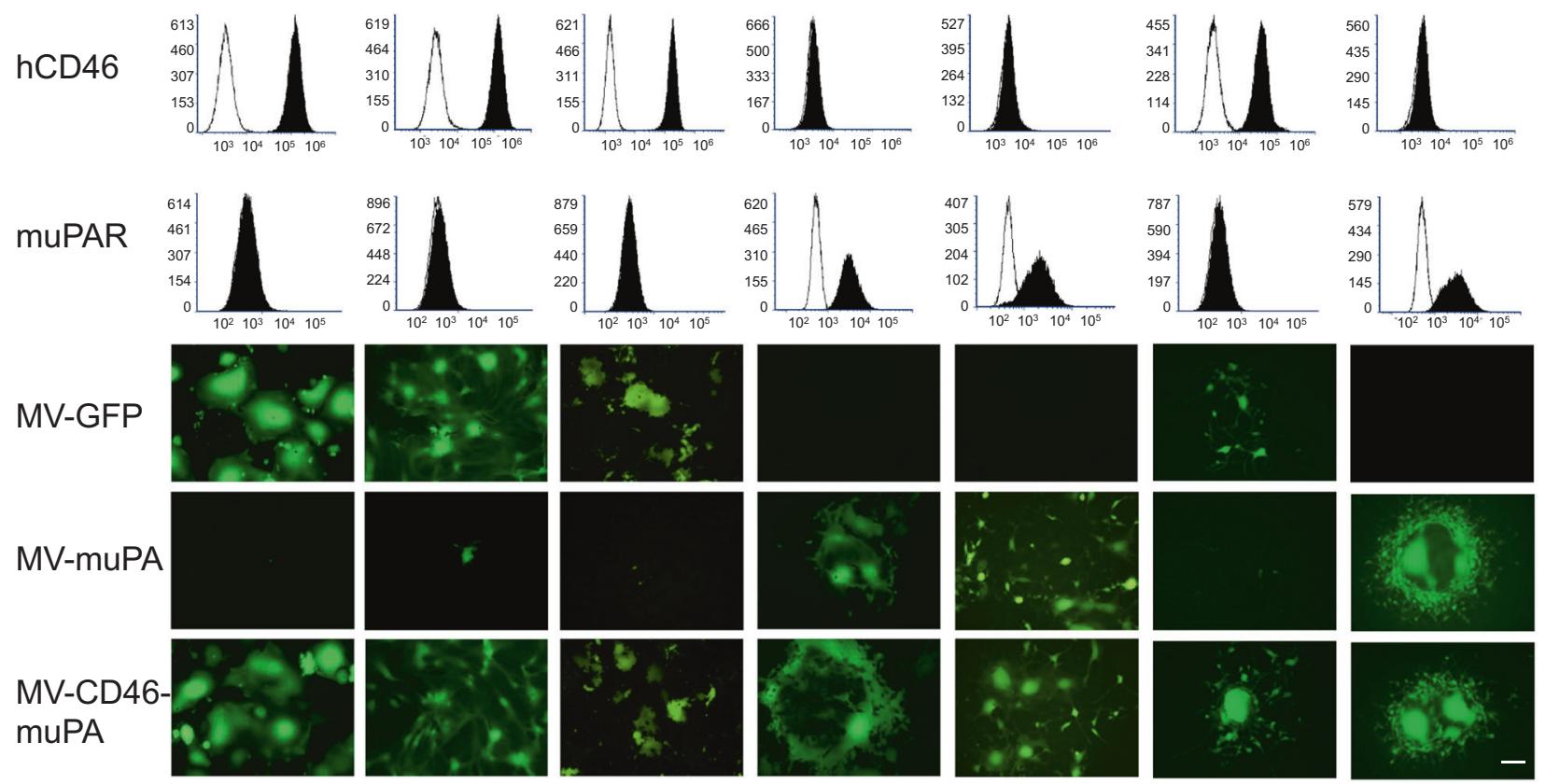

Fig. 1 Characterization of dual targeting MV-CD46-muPA. a MVCD46-muPA was generated by displaying the amino terminal fragment (ATF) of murine uPA (flanked by the Sfil/NotI restriction sites) as a C-terminal extension of unmodified MV-H glycoprotein, as in methods. b In vitro tumor and species specificity of MV-GFP, MVmuPA, and MV-CD46-muPA. Human CD46 (hCD46) and mouse uPAR expression were detected by FACS. Human cancer cells (MDA-

MB-231, 786-O, HT-29), human cancer associated fibroblast CAF23, murine cancer cells (4T1, CT-26), and murine fibroblast 3T3 cells were infected with MV-GFP, MV-muPA, or MV-CD46-muPA at an $\mathrm{MOI}=1$ and photographed $48 \mathrm{~h}$ after infection using a fluorescent microscope. Pictures are representative of independent experiments performed in triplicate. Scale bar $=100 \mu \mathrm{m}$.

modulators of tumor-stromal interactions $[11,16]$. Targeting the stromal barrier in an experimental model is particularly difficult with the MV platform, owing to the fact that MV does not naturally infect murine tissues; therefore, the great majority of murine models using nontargeted or retargeted MVs have not been able to assess the effects of MVs on the tumor microenvironment.

We have previously developed and characterized novel, species specific oncolytic MVs fully retargeted against the human or murine urokinase receptor (UPAR) [17, 18], a GPI-anchored cell surface receptor, which is overexpressed in tumor and particularly in stromal cells, and whose role in tumor-stromal interactions and cancer progression are well established [19-25]. We demonstrated that systemic 
administration of species specific, human (MV-huPA) or murine (MV-muPA) uPAR retargeted MVs is safe, successfully targets tumor tissues over non-cancer tissues, and is associated with significant tumor delaying effects in primary or metastatic, xenograft, and syngeneic cancer models, respectively $[18,26]$. Taking advantage of the species specificity of MV-muPA, we recently reported that (murine) stromal-selective MV-muPA was associated with direct stromal targeting in a human breast cancer xenograft (where human cancer cells are not permissive to the murine targeted virus), leading to modulation of murine stromal gene expression, indirect effects on tumor cell gene expression, and measurable in vivo tumor growth delay by MV-muPA [27]. However, the in vivo antitumor effects of targeting tumor-stromal cells alone were modest compared to tumor selective targeting, suggesting that combined tumor and stromal targeting may further enhance antitumor efficacy.

In order to understand the effects of combined stromal and tumor targeting vs. tumor targeting alone by an $\mathrm{OV}$ in an experimental system, the viral vector should be able to target each component independently, via different receptors. Using a model where stroma and tumor cells are of different species would further facilitate comparative assessment of combined vs. single (tumor) targeted OVs. To achieve this, we engineered MV-CD46-muPA, a novel, dual species, dual targeting oncolytic MV. This viral vector has the ability to target both murine cells, via species specific murine uPAR, and human cancer cells, via human CD46 (and not recognized by mouse cells). In this report, we characterize the differential in vitro, in vivo, and biological effects of combined tumor and stromal vs. tumor (via CD46) only targeting by oncolytic MVs in experimental cancer models.

\section{Materials and methods}

\section{Virus engineering and propagation}

Engineering and rescue of MV-GFP and MV-muPA, propagation, and titration were previously reported [15, 17]. To generate MV-CD46-muPA, the amino terminal fragment (ATF) of mouse urokinase was amplified by PCR from pcDNA3.1(+)-muPA [28], cloned into the SfiI and NotI cloning sites of pTNH6 [29], a non-modified MV H glycoprotein expression construct, to create pTNH-m-ATF, and verified by DNA sequencing. MV-H glycoproteins from pTNH-m-ATF were inserted into the PacI and SpeI sites of $\mathrm{p}(+) \mathrm{MV}$-eGFP, which encodes the full-length infectious clone of MV Edmonston tag strain and carries the eGFP gene [15] (Fig. 1a), resulting in the p(+)MV-CD46muPA construct (Fig. 1a). The MV-CD46-muPA virus was rescued using previously reported methods [29].

\section{Cell culture}

MDA-MB-231 (ATCC \#HTB-26, human breast cancer), 786O (ATCC \#CRL-1932, human renal cancer), HT-29 (ATCC \#HTB-38, human colon cancer), 4T1 (ATCC \#CRL-2539, murine mammary carcinoma), CT-26 (ATCC \#CRL-2639, murine colon cancer) and NIH-3T3 (ATCC \#CRL-1658, immortalized mouse fibroblasts) cells were purchased from the American Type Culture Collection (ATCC, Manassas, VA) and were authenticated. Cells were maintained in Dulbecco's modified Eagle's medium (DMEM) containing 10\% fetal bovine serum (FBS), penicillin, and streptomycin. CAF23 tumor-associated fibroblasts were isolated from primary breast tumors [30], a generous gift from Dr. Doraya ElAshry, and maintained in IMDM medium. Vero- $\alpha$ His cells [15] were grown in DMEM containing 10\% FBS. All the cell lines were grown under mycoplasma-free conditions at $37^{\circ} \mathrm{C}$, $5 \% \mathrm{CO}_{2}$.

\section{In vitro viral infection, cytotoxicity, and in vitro viral replication}

In vitro viral infection, cytotoxicity, and in vitro viral replication were performed as previously described $[17,18]$. In brief, Cells were plated in six-well plates at a density of $10^{5}$ per well. Twenty-four hours after seeding, the cells were infected at MOI $=1$ in $1 \mathrm{~mL}$ of Opti-MEM for $2 \mathrm{~h}$ at $37^{\circ} \mathrm{C}$. At different time points after infection, the number of viable cells (determined by tryptan blue exclusion) in each well was counted using Vi-Cell cell viability analyzer (Beckman Coulter, Fullerton, CA). Results are shown as percent of viable cells, compared with untreated control, which was calculated by dividing the number of viable cells in the infected well by the number of viable cells in the uninfected well. For in vitro viral replication assay, each cell line (in duplicate) was infected with $\mathrm{MV}$ at an MOI of 3 and incubating at $37^{\circ} \mathrm{C}, 5 \% \mathrm{CO}_{2}$ for $2 \mathrm{~h}$, after which the virus was removed, and cells were maintained in $5 \% \mathrm{FBS}$ of DMEM at $37^{\circ} \mathrm{C}$. Virus titers were obtained by titration on Vero- $\alpha$ His cells and expressed as $50 \%$ tissue culture infectious dose $\left(\mathrm{TCID}_{50}\right) / \mathrm{ml}$ at the specified time points.

\section{In vitro fibroblast to cancer cell viral transfer}

Fibroblasts (CAF23, NIH-3T3) were incubated with species specific uPAR retargeted MVs, which express eGFP (MOI of 1) in Opti-MEM for $2 \mathrm{~h}$ at $37^{\circ} \mathrm{C}$. Free viruses were then removed by $\mathrm{PBS}$ washing and cells were maintained in the appropriate medium. To rule out persistence of cell free virions, $100 \mu \mathrm{l}$ of the last wash was inoculated on Vero$\alpha$ His monolayers. Infected cells were cultured in the presence of $80 \mu \mathrm{g} / \mathrm{ml}$ fusion inhibitory peptide (FIP sequence: Z-D-Phe-Phe-Gly-OH, Bachem, Torrance, CA) to block 
syncytia formation during overnight incubation. Virus infected fibroblasts were then washed before overlay on RFP-expressing tumor cells. Mixed (double-color) syncytia were demonstrated at different periods of incubation by fluorescent microscopy. Cells were incubated with eFluor780 (Fixable viability dye, ebioscience) for $10 \mathrm{~min}$ at $4{ }^{\circ} \mathrm{C}$ and washed twice in fluorescence-activated cell sorting (FACS) buffer. Cells were collected and analyzed using CytoFLex (BD Biosciences) for quantification of viable RFP-expressing tumor cells.

\section{Animal studies}

Animal studies were approved by the University of Miami Institutional Animal Care and Use Committee. All methods were performed in accordance with relevant guidelines and regulations, and proper sample size was calculated. Eight to 10 -week-old female NSG mice (Jackson labs, Farmington, CT) were injected with $2 \times 10^{6}$ HT-29 cells subcutaneously into the left flank. When tumors reached a volume of $30-60 \mathrm{~mm}^{3}$, mice ( $n=8$ /group) were randomly divided into three groups, and treated with PBS, $1.5 \times 10^{6} \mathrm{TCID}_{50}$ of MV-GFP, or MV-CD46-muPA via tail vein three times every other day. Tumor measurements were performed with proper blinding twice a week and calculated with the following formula (width ${ }^{2} \times$ length $\times 0.5$ ) as reported [17]. Clinical signs of toxicity were monitored. Animals were followed until they reached kill criteria (when tumor burden reached $10 \%$ of body weight, tumor ulceration occurred or mice became moribund).

\section{Terminal deoxynucleotidyl transferase dUTP nick end labeling (TUNEL) assay}

To detect and visualize apoptosis, slides were washed twice with PBS (after fixation), permeabilized with $0.2 \%$ TritonX100 for $20 \mathrm{~min}$ at room temperature and after two additional washes with PBS, sections were probed with label solution (for negative controls) or TUNEL reaction mix, following manufacturer's instructions (In Situ Cell Death Detection Kit-TMR red; Roche Applied Science, Indianapolis, IN) $[18,26]$.

\section{Immunohistochemistry and immunofluorence studies}

Tumor-bearing mice $(n=3)$ were randomly divided into three groups and treated with two intravenous injections of PBS, $1.5 \times 10^{6} \mathrm{TCID}_{50}$ of MV-GFP or MV-CD46-muPA. Tumor samples were collected and frozen, and cryostat sections were fixed in cold acetone for $10 \mathrm{~min}$ and endogenous peroxidase activity were quenched with $0.3 \% \mathrm{H}_{2} \mathrm{O}_{2}$ for $10 \mathrm{~min}$. The slides were washed in PBS and incubated with anti-muPAR antibody (R\&D Systems, \#AF534, Minneapolis, MN) for $30 \mathrm{~min}$ at $37^{\circ} \mathrm{C}$. After washing in PBS, the slides were developed with Anti-Goat HRP-DAB Cell \& Tissue Staining Kit (brown; R\&D Systems, \#CTS008) and counterstained with hematoxylin (blue) according to the manufacturer's instructions.

Cryostat sections were fixed in cold acetone for $10 \mathrm{~min}$. The slides were washed in PBS and stained with anti-MVnucleoprotein-FITC antibody (Chemicon International, \#MAB8906F, Temecula, CA), anti-Ki67 antibody-FITC (Thermo Fisher Scientific, \#11-5698-82, Waltham, MA), anti-CD140a antibody (Cell signaling, \#3174), anti-CD31 antibody (Abcam, \#ab28364) or anti-CD105 antibody (Abcam, \#ab107595) with Alexa fluor 488 anti-rabbit IgG. Slides were mounted with anti-fade mounting medium after three PBS washes and analyzed by fluorescent microscopy.

\section{Analysis of tumor and stromal cells by flow cytometry}

For in vitro studies, cells were harvested by cell dissociation buffer (Invitrogen, Carlsbad, CA), washed twice in ice-cold phosphate-buffered saline, and then the surface expression of relevant receptors was detected using anti-mouse UPAR PE antibody (R\&D systems, \#FAB531P, Minneapolis, MN), and anti-human CD46 PerCP-eFluor710 antibody (eBioscience, \#46-0469-42, San Diego, CA). Washed cells were analyzed using CytoFLex (BD Biosciences) and FCS Express software (De Novo Software, Pasadena, CA). For in vivo studies, HT-29 tumor-bearing mice ( $n=3$ /group) were randomized into three groups and treated with two intravenous injections of vehicle, $1.5 \times 10^{6} \mathrm{TCID}_{50}$ of MVGFP or MV-CD46-muPA. Fourteen days after MV treatment, mice were killed and tumors were excised. To prepare tumor cell suspensions, tumor tissues were dissected and sectioned into small fragments, followed by digestion with $1 \mathrm{mg} / \mathrm{mL}$ of collagenase in complete RPMI media $(10 \%$ FBS, $1 \%$ penicillin, streptomycin) prior to using Gentle MACS Dissociator. Cell suspension was passed through a $70-\mu \mathrm{m}$ nylon strainer to obtain a single cell population. Cells were incubated with eFluor780 (Fixable viability dye, ebioscience) for $10 \mathrm{~min}$ at $4{ }^{\circ} \mathrm{C}$ and washed twice in FACS buffer prior to staining in order to distinguish live from dead cells. Cells were then incubated with Fc block for $20 \mathrm{~min}$ at $4{ }^{\circ} \mathrm{C}$ and incubated for $30 \mathrm{~min}$ at $4{ }^{\circ} \mathrm{C}$ with the following antibodies: CD45 APC/CY7 (Bio Legend \#368516), CD140 APC (Bio Legend, \#135908), murine uPAR PE (R\&D Systems, \#FAB531P), murine CD105 PE/CY7(Bio Legend, \#120410), and murine CD31 Pacific blue (Bio Legend, \#102422). Stained cells were washed by FACS 
a 1

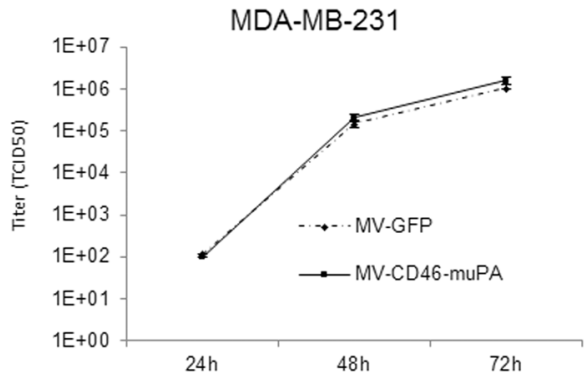

III

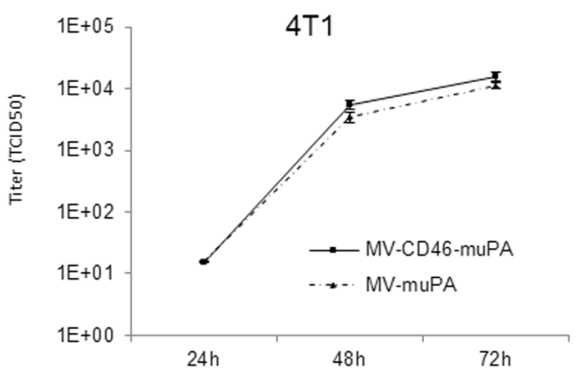

b

I

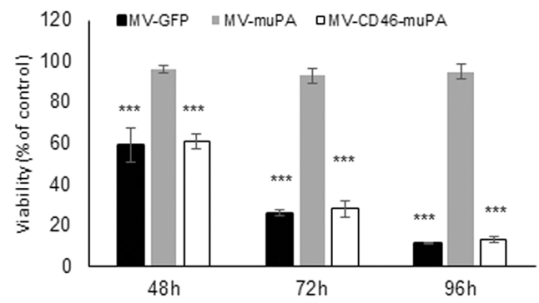

III

$4 T 1$

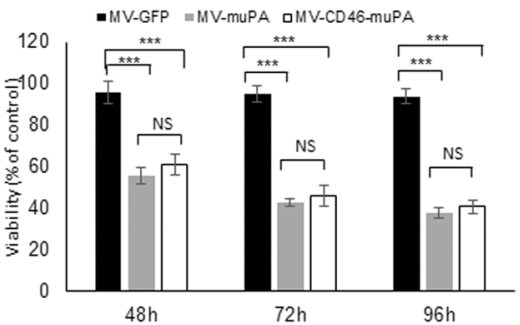

Fig. 2 In vitro replication and cytopathic effects of MV in human and murine cancer cells. a In vitro replication. Human cancer cells MDA-MB-231 (a, I), HT-29 (a, II) were infected with MV-GFP, and MV-CD46-muPA (MOI = 3). Murine cancer cells 4T1 (a, III) and CT26 (a, IV) were infected with MV-muPA, and MV-CD46-muPA $(\mathrm{MOI}=3)$. Titers of virus were determined at different time points by the one-step growth curve (duplicate experiments). b In vitro cytopathic effects. Human cancer cells MDA-MB-231 (b, I), HT-29 (b, II)

buffer to remove unbound antibodies and plated for flow cytometric analysis using CytoFLex (BD Biosciences). In total, 200,000 events (viable cells) per sample were counted and normalized for grams of tissue for graphical display and comparison.
II
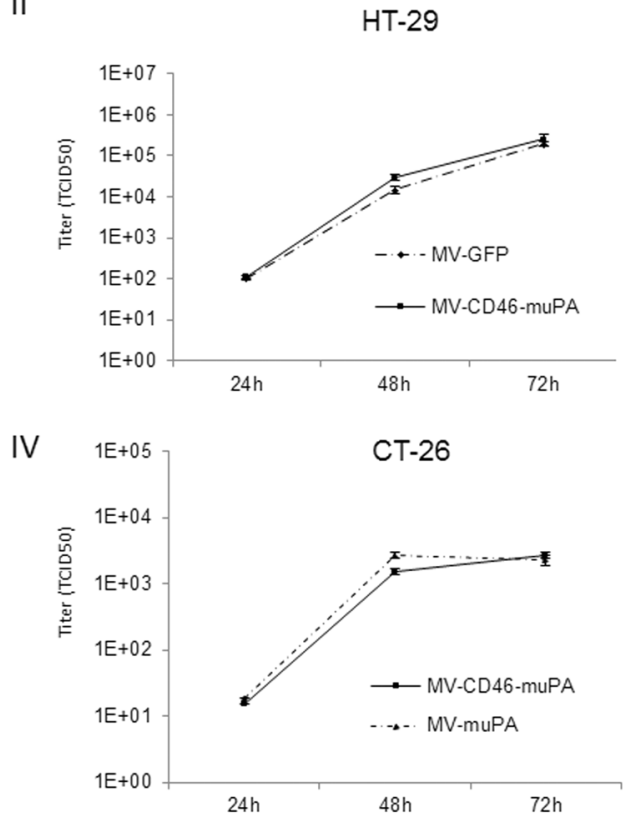

II

HT-29

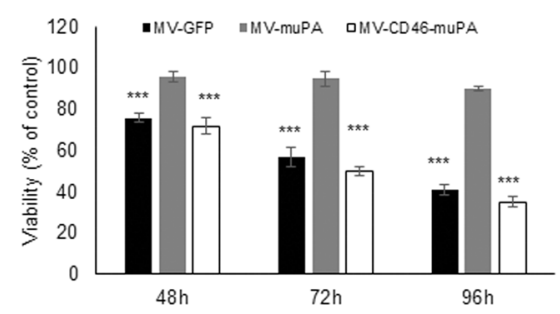

IV

CT26

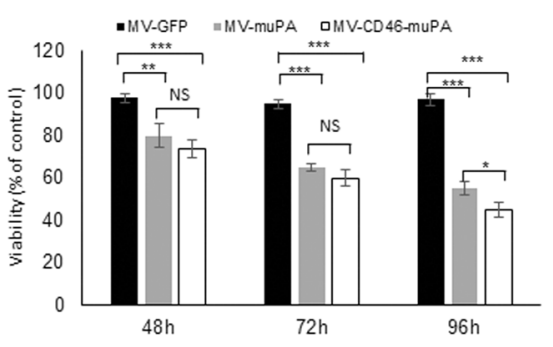

were infected with MV-GFP, MV-muPA, and MV-CD46-muPA $(\mathrm{MOI}=1), * * p<0.01, * * * p<0.0001$ (MV-muPA vs MV-GFP, MVmuPA vs MV-CD46-muPA). Murine cancer cells 4T1 (b, III) and CT26 (b, IV) were infected with MV-GFP, MV-muPA and MV-CD46muPA $(M O I=1)$. Viability was determined at $48 \mathrm{~h}, 72 \mathrm{~h}$, and $96 \mathrm{~h}$ by trypan blue exclusion and presented as percent of controls. Bars represent averages $\pm \mathrm{SD}$ of triplicate experiments. $* p<0.05$; $* * p<$ $0.01, * * * p<0.0001$ (Tukey-Kramer test). NS: not significant.

\section{RPPA analysis of tumor samples}

Functional proteomic (human proteins) analysis of tumor samples was performed at the RPPA Core Facility at MD Anderson Cancer Center (Houston, TX), using tumor 
Fig. 3 Effects of viral fibroblast targeting on fibroblast-tumor cell interactions in vitro. Murine 3 T3 fibroblasts were infected with MV-GFP, MV-muPA, and MV-CD46-muPA (MOI $=1)$ as in methods, and then overlaid on RFP-expressing HT-29 (a, I) or MDA-MB-231 (b, I) cancer cells. Mixed (double-color) syncytia were demonstrated at $48 \mathrm{~h}$ incubation by fluorescent microscopy. In a similar way, MV-GFP, MV-muPA, and MVCD46-muPA $(\mathrm{MOI}=1)$ infected CAF23 cells were overlaid on RFP-expressing murine 4T1 (c, I) or CT-26 (d, I) cells and yellow syncytia were demonstrated at $48 \mathrm{~h}$ incubation by fluorescent microscopy. Scale bar $=100 \mu \mathrm{m}$. Effects of fibroblast specific viral targeting on cancer cell growth in vitro $72 \mathrm{~h}$ after overlay. HT-29 (a, II) and MDA-MB-231 (b, II), 4T1 (c, II) or CT-26 (d, II) Bars represent averages $\pm \mathrm{SD}$ of triplicate experiments. $* p<0.05$; $* * p<0.01$. $* * * p<0.0001$ (Tukey-Kramer test). NS: Not significant. a

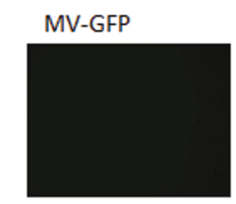

MV-muPA
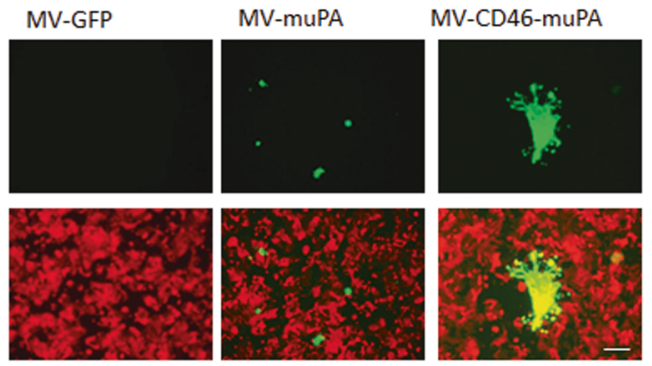

b ।

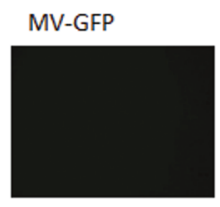

MV-muPA
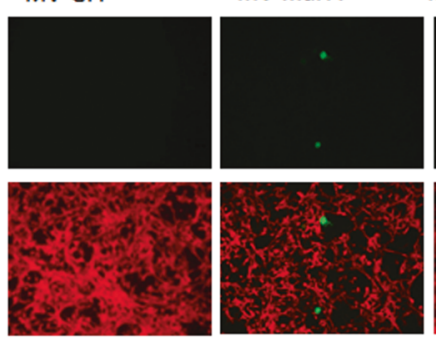

c
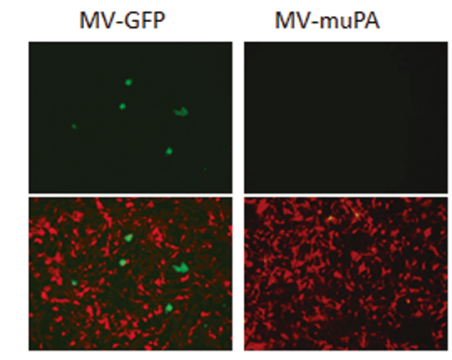

d

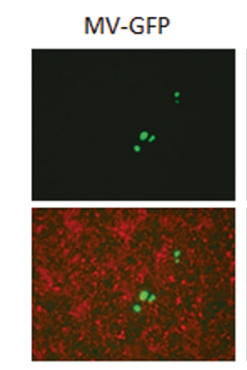

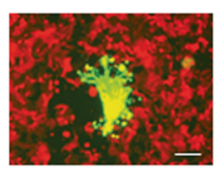
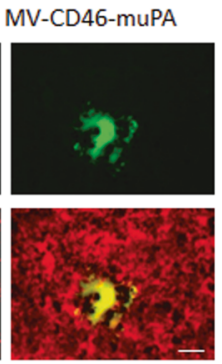

MV-CD46-muPA
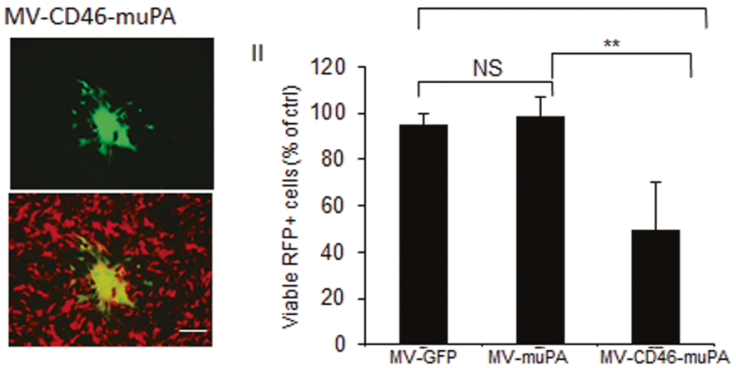

II

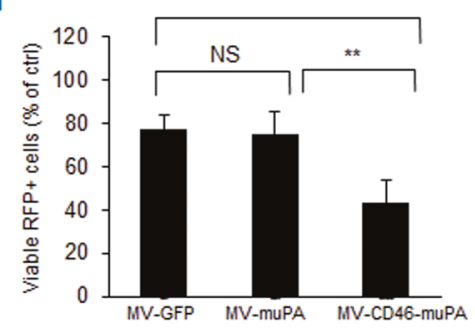

II

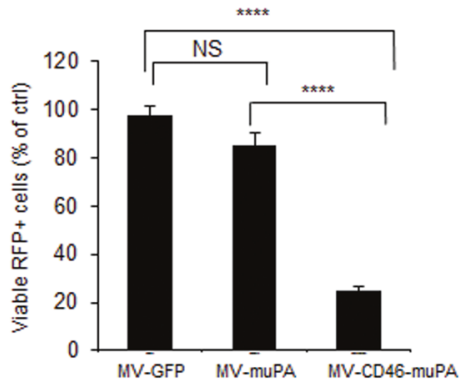

MV-CD46-muPA
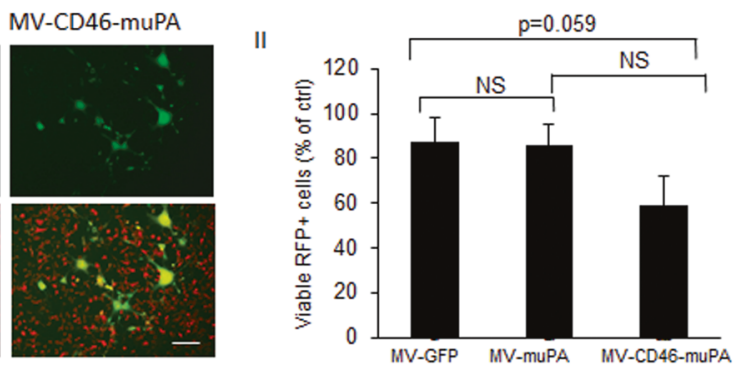

lysates from vehicle and virus (MV-GFP and MV-CD46muPA) treated HT-29 tumors, resected at day 10 after last virus treatment. Antibodies and approaches are described at the RPPA website (https://www.mdanderson.org/research/ research-resources/core-facilities/functional-proteomicsrppa-core.html). RPPA data generation and normalization (in $\log 2$ scale) were handled and performed by RPPA Core facility at MD Anderson Cancer Center. Differential expression analysis for comparisons between MV-GFP- or MV-CD46-muPA-treated and control samples was performed using a linear model with $\mathrm{R}$ package "limma" (v3.26.9) [31], and significance was evaluated with false discovery rate (FDR) [32]. Proteins with $p$ value $<0.05$ and FDR $<0.2$ were analyzed for pathway enrichment using Ingenuity Pathway Analysis (IPA, QIAGEN Redwood City, www.qiagen.com/ingenuity), and were categorized according to Disease and Bio Functions. All RPPA data accompanying the pre-calculated analytic results are included in supplementary data.

\section{Statistical analysis}

In vitro data are presented as means $\pm \mathrm{SD}$. Results from in vivo studies are shown as means \pm SEM. All in vitro experiments were performed in triplicate unless otherwise specified and repeated at least twice. Statistical analysis among groups was performed by ANOVA, and sub-group comparisons were made with the Tukey-Kramer test, as appropriate. Overall survival was analyzed by the Kaplan-Meier method and differences were analyzed by the 


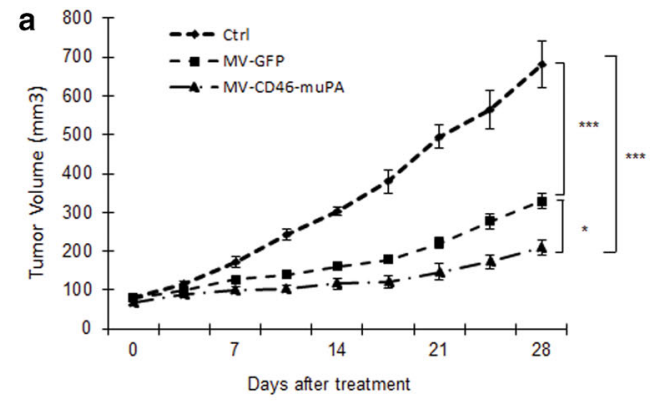

C I
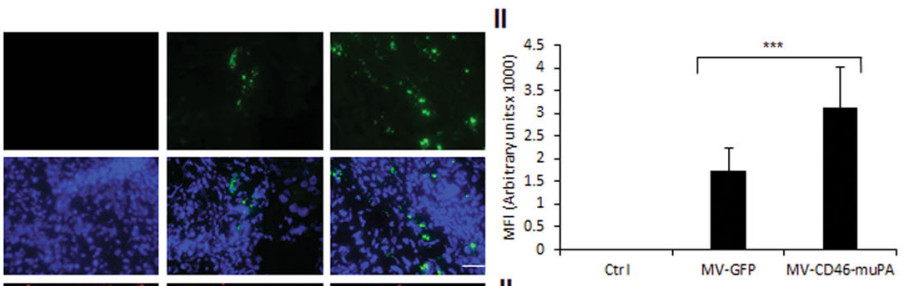

d
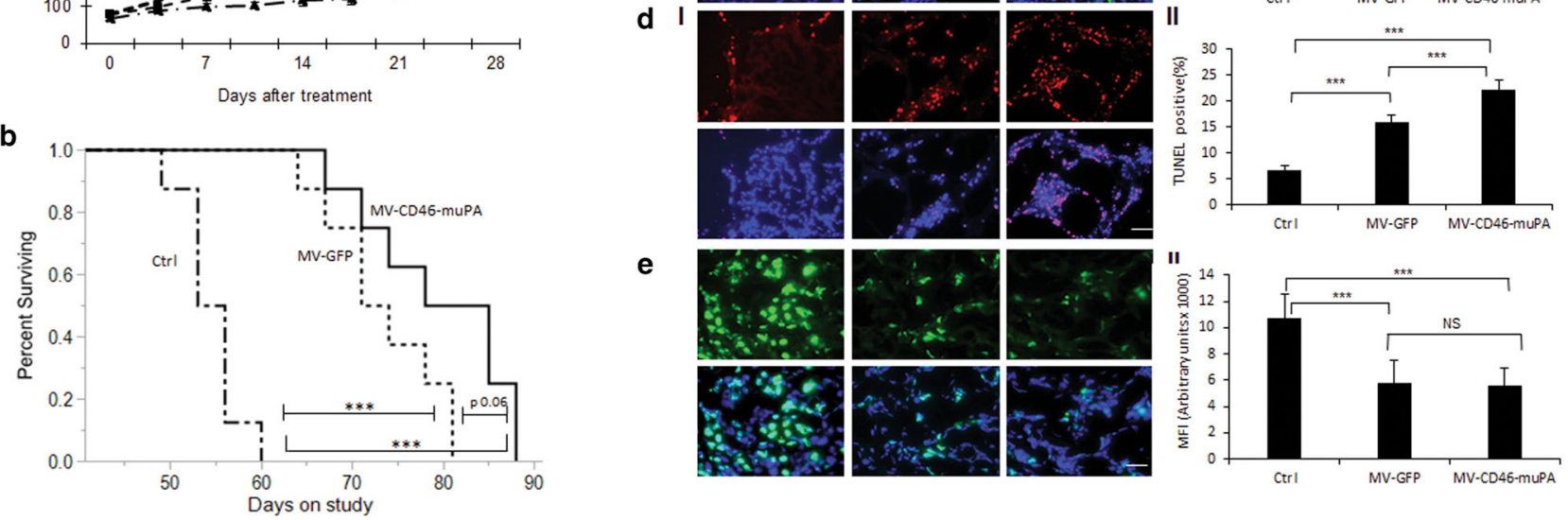

Fig. 4 In vivo effects of MV-CD46-muPA on human colon cancer xenografts. a Effects of MV-GFP and MV-CD46-muPA on HT-29 tumor progression. Tumor-bearing NSG mice ( $n=8$ per group) were treated with vehicle (PBS) or MV-GFP and MV-CD46-muPA intravenously, and tumor volume was followed as in methods.. ${ }^{*} p<0.05$; $* * p<0.01 ; * * * p<0.001$ (days 21, 25, and 28; Tukey-Kramer test). NS: Not significant. b Kaplan-Meier analysis of survival of HT-29 tumor-bearing mice treated with vehicle, MV-GFP, or MV-CD46muPA. Mice were monitored until they reached killing criteria. ${ }^{*} p<$ $0.05 ; * * p<0.01$. $* * * p<0.001$ ((log-rank test). NS: Not significant. c Stromal targeting by MV-m-uPA. HT-29 tumor-bearing NSG mice

log-rank test. Statistical significance was set at $* p<0.05$, $* * p<0.01, * * * p<0.001$, with adjustments for multiple comparisons as appropriate. $P$ value $<0.05$ were considered statistically significant. All statistical tests were two-sided.

\section{Results}

\section{In vitro rescue and characterization of MV-CD46- muPA, a dual targeted oncolytic MV}

We have previously demonstrated the in vitro and in vivo abilities of species specific oncolytic MVs fully retargeted against either human or murine uPA receptor [17]. To evaluate the contribution of stromal and tumor targeting on MV's overall antitumor effects, a dual targeted oncolytic MV (MV-CD46-muPA), able to bind human cancer cells via CD46 and murine cells via murine uPAR targeting, was engineered and rescued (see methods and Fig. 1a). Comparative analysis of virus infection among the unmodified MV-GFP (targeting CD46 only, human cells), murine UPAR retargeted MV-muPA (targeting mouse cells expressing muPAR only) and MV-CD46-muPA (targeting were treated with either vehicle, MV-GFP and MV-CD46-muPA. Immunofluorescence staining for MV-N (green) in tumor tissues obtained at day 3 after virus administration (c, I). Scale bar $=20 \mu \mathrm{m}$. d, e. Representative pictures of TUNEL (d, I), and Ki67 (e, I) antibody staining of treated and untreated tumors from mice at day 6 after treatment. Scale bar $=20 \mu \mathrm{m}$. Analysis of MV-N (c, II), TUNEL-positive nuclei (d, II), and Ki67 (e, II) staining in vehicle, MV-GFP and MV-CD46-muPA-treated tumors. Bars represent averages \pm SEM of triplicate experiments ( $n=3$ per group). $* p<0.05$; $* * p<0.01 ; * * * p<0.001$ (days 21, 25, and 28; Tukey-Kramer test).

murine and human cells) showed that only the dual targeted virus successfully infected both human and mouse cancer cells, whereas MV-GFP and MV-muPA-infected cells in a species specific manner (Fig. 1b). In addition, MV-CD46muPA successfully replicated (Fig. 2. A) and induced cytotoxicity (Fig. 2b) in murine and human cells, whereas single targeted viral vectors induced oncolysis in species specific cell lines only. Titers of MV-GFP in infected mouse cancer cells, and titers of MV-muPA in infected human cancer cells were undetectable (data not shown). The above results confirmed the murine and human targeting ability of MV-CD46-muPA.

\section{In vitro stromal-to-tumor and tumor-to-stromal viral transfer by MV-CD46-muPA}

We next assessed the in vitro ability of virus infected stromal cells to transfer viral infection to tumor cells and vice versa. Towards this goal, in vitro mouse-human coculture assays were performed, where 3T3 murine fibroblasts were infected by MV-muPA, MV-GFP, or MV-CD46-muPA, and after overnight incubation, overlaid onto RFP-expressing human cancer cells. Successful 

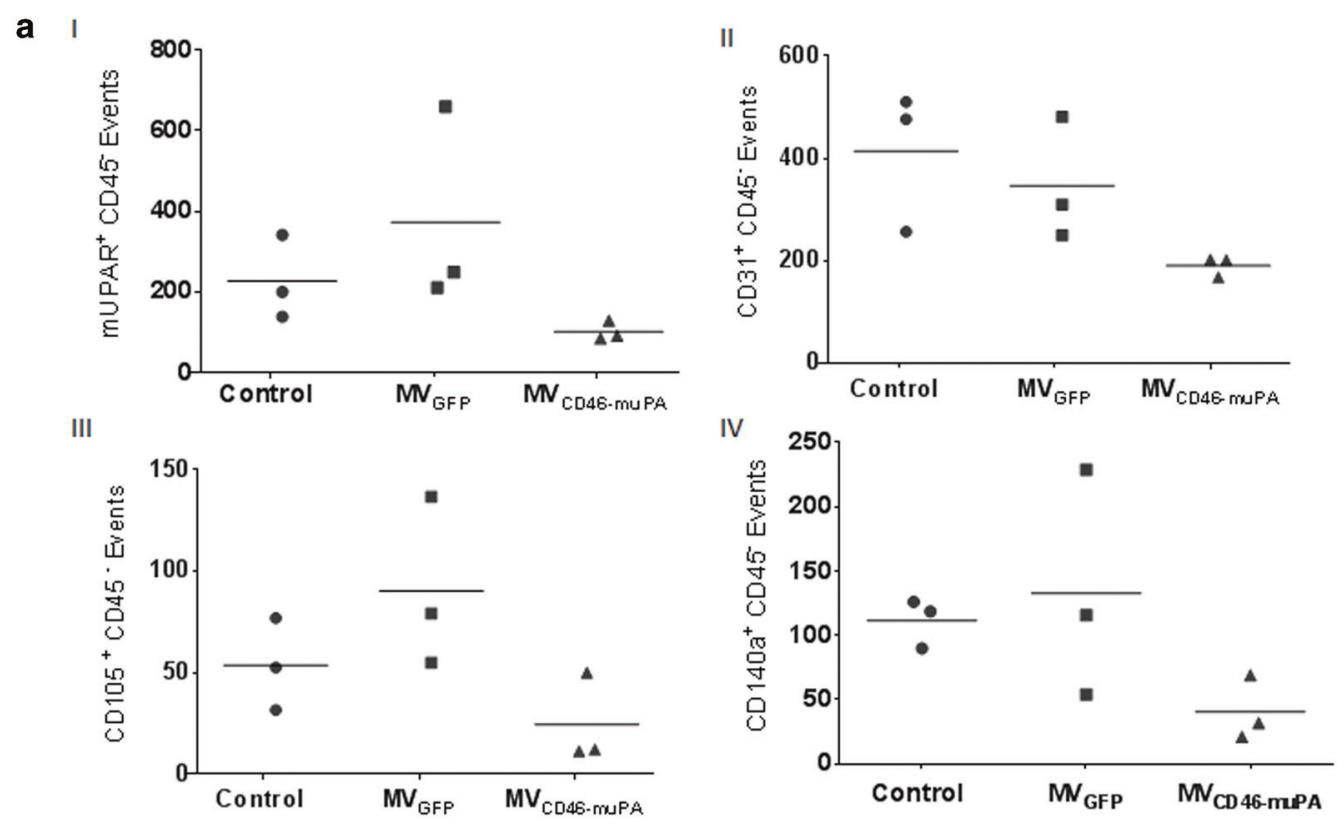

b

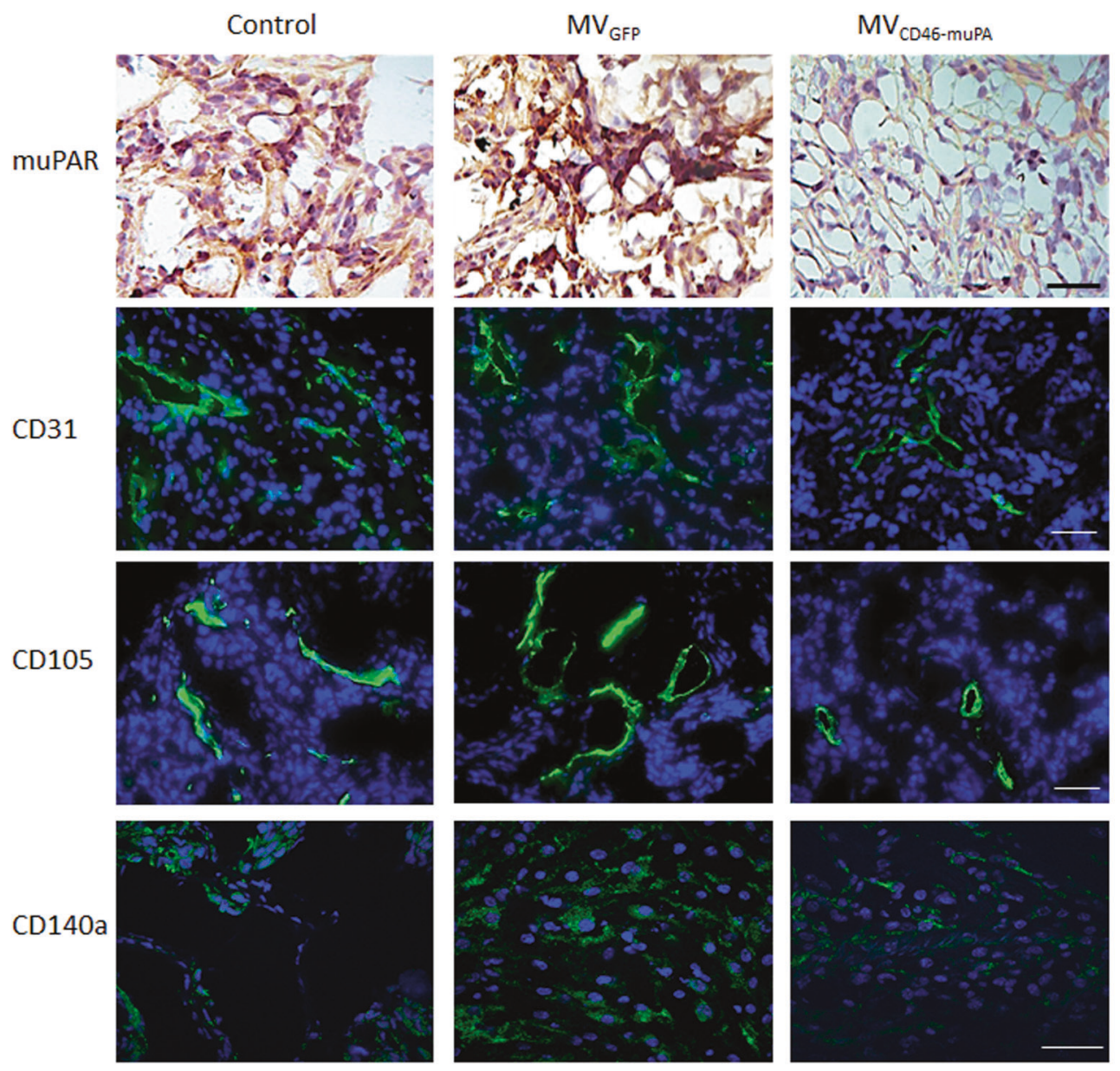

stromal to tumor cell viral transfer was detected by the presence of yellow fluorescent syncytia in merged (GFP + RFP) pictures. These findings are in line with the findings by Castleton et al. [33], who previously demonstrated virus transfer from infected mesenchymal stem cells to leukemia cells in the presence of neutralizing antibodies. As shown in 
Fig. 5 In vivo effects of virus treatment on tumor stroma. HT-29 tumor-bearing mice ( $n=3$ /group) were treated with two intravenous injections of vehicle, $1.5 \times 10^{6} \mathrm{TCID}_{50}$ of MV-GFP or MV-CD46muPA. Tumors (three per group) were excised and processed for flow cytometric analysis as described in methods. Data are displayed as graphical quantification of events per gram of tumor (iv) for (a, I) CD45- muPAR + , (a, II) CD45- CD31 +, (a, III) CD45- CD105 +, and (a, IV) CD45- CD140a + cells. b Representative pictures of muPAR, CD31, CD105, and CD140a antibody staining of treated and untreated tumors ( $n=3$ per group). Scale bar $=20 \mu \mathrm{m}$.

Fig. 3a, b, successful murine fibroblast to tumor cell viral transfer was observed only in cells treated with MV-CD46muPA but not with the single targeted viruses, as demonstrated by identification of yellow (GFP and RFP) fluorescent syncytia on merged pictures. Moreover, tumor cell cytotoxicity (measured by flow cytometric quantification of viable RFP tumor cells) was significantly increased by infection of MV-CD46-muPA compared to MV-GFP or MV-muPA (Fig. 3a.II, 3. b. II). In a similar manner, successful human CAFs to RFP-expressing murine cancer cell viral transfer was observed in co-cultures treated with the dual, but not the single targeted MVs (Fig. 3c.I, 3. d. I), resulting in significant murine cancer cell cytotoxicity (Fig. 3c. II; 3d. II).

\section{In vivo antitumor effects of dual vs single targeted oncolytic MVs}

Next, to validate the above findings in the in vivo setting, mice bearing human HT-29 (colon adenocarcinoma) tumors were treated with three intravenous administrations of vehicle, MV-GFP (targeting CD46 only) and dual targeted MV-CD46-muPA. Animals were followed for tumor progression and survival. As shown in Fig. 4a, while both MVGFP and MV-CD46-muPA-treated mice had improved outcomes compared with vehicle-treated controls, there was a clear improvement in antitumor activity in the group of mice treated with the dual targeted virus (Fig. 4a, b). Tumors treated with MV-CD46-muPA had a 69\% reduction in average tumor volume compared with control mice and $37 \%$ reduction compared with MV-GFP-treated mice at day 25 of treatment (Fig. 4a). Median survival was also improved in mice treated with the dual targeted virus compared with controls and to MV-GFP (Fig. 4b). No significant treatment related toxicity/treatment related deaths were observed in treated vs control groups.

\section{Correlative tumor studies}

To determine the mechanisms explaining the in vivo differential effects between single vs. dual targeted oncolytic MVs, 3 tumor (HT-29) bearing mice per group were treated with intravenous vehicle, MV-GFP or MV-CD46-muPA as in methods. Tumors were resected at day 6 after the last virus treatment. Successful tumor targeting was demonstrated by immunofluorescence analysis of viral protein (MV-N), with significantly higher levels observed in tumors treated with the dual, compared with the single targeted oncolytic MV (Fig. 4c. I, II). Next, staining for proliferation (Ki67) and apoptosis (TUNEL) markers were performed. Although both MV-GFP- and MV-CD46-muPA-treated tumors were associated with significantly decreased tumor cell proliferation and increased apoptosis, tumors treated with the dual targeted viruses were associated with more significant apoptosis compared with MV-GFP in the HT-29 (Fig. 4d).

To determine the direct in vivo effects of MV-CD46muPA on murine stromal cells, additional HT-29 tumorbearing mice were treated as above, tumors resected and processed for flow cytometric analysis at day 14 post-treatment, a time where tumor volume curves clearly separated, to evaluate viable tumor fibroblasts $(\mathrm{CD} 45(-) / \mathrm{CD} 140 \mathrm{a}(+)$ [34], tumor endothelial cells $[(\mathrm{CD} 45(-) / \mathrm{CD} 31(+)$ and CD45 $(-) / \mathrm{CD} 105(+)]$, and CD45-/muPAR $(+)$ cells. At this time point, murine $\mathrm{uPAR}+$ (stromal) cells were decreased in the dual targeted virus treated groups compared with vehicle (average 55\% decrease) or MV-GFP (73\% decrease)-treated groups (Fig. 5a, I). CD31-positive endothelial cells were also decreased in the dual targeted treated tumors (54\% and $45 \%$ decrease compared with vehicle and MV-GFP, respectively, (Fig. 5a, II), as were CD105+endothelial cells (54\% and 73\% decrease compared with vehicle and MV-GFP, respectively (Fig. 5a, III). Tumor fibroblasts (CD45-/CD140a(+) were also noticeable decreased in the MV-CD46-muPA-treated group compared with vehicle (64\% decrease) or MV-GFP (69\% decrease)-treated tumors (Fig. 5, IV). These observations were further validated by immunohistochemistry (muPAR) and immunofluorescence (CD31, CD105, CD140a) studies of tumor from treated and untreated mice (Fig. 5b)

To gain further insight into the mechanisms of in vivo antitumor activity of single vs. dual targeted oncolytic MVs in the HT-29 model, functional proteomic analysis (RPPA) was performed as in methods, from tumors resected from treated mice at day 10 after virus treatment. Figure 6. A shows pathway analysis (as a heat map) of differentially expressed (human) proteins involved in cell cycle, development, growth, proliferation, as well as cell death and survival pathways. Selected up- and downregulated proteins modulated by MV-GFP or MV-CD46-muPA are shown in Fig. 6b. Both MV-GFP and MV-CD46-muPA-treated tumors showed increased expression of the tumor suppressor proteins PTEN and Merlin, as well as significant downregulation of the transcription factors or transcriptional co-activators GATA-6, ELK-1, SOX-2, and Yap (Fig. 6b). Moreover, MV-CD46-muPA (but not MV-GFP) induced downregulation of LDH-A, hexokinase, GCLM, TFRC, 
a

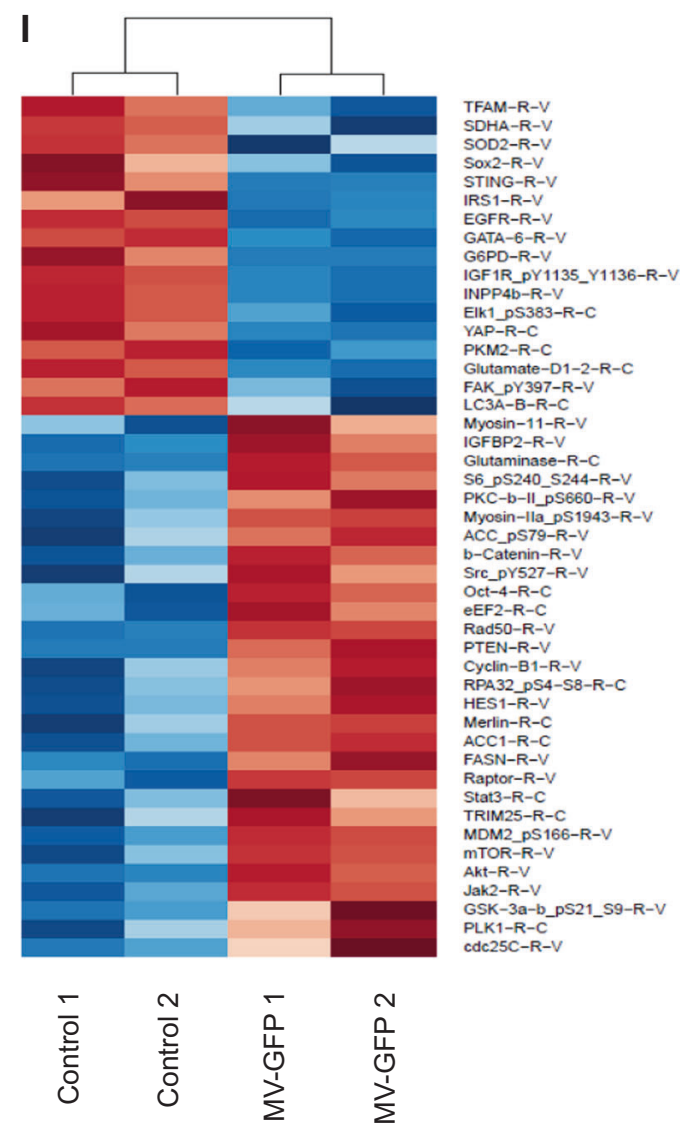

\section{II}
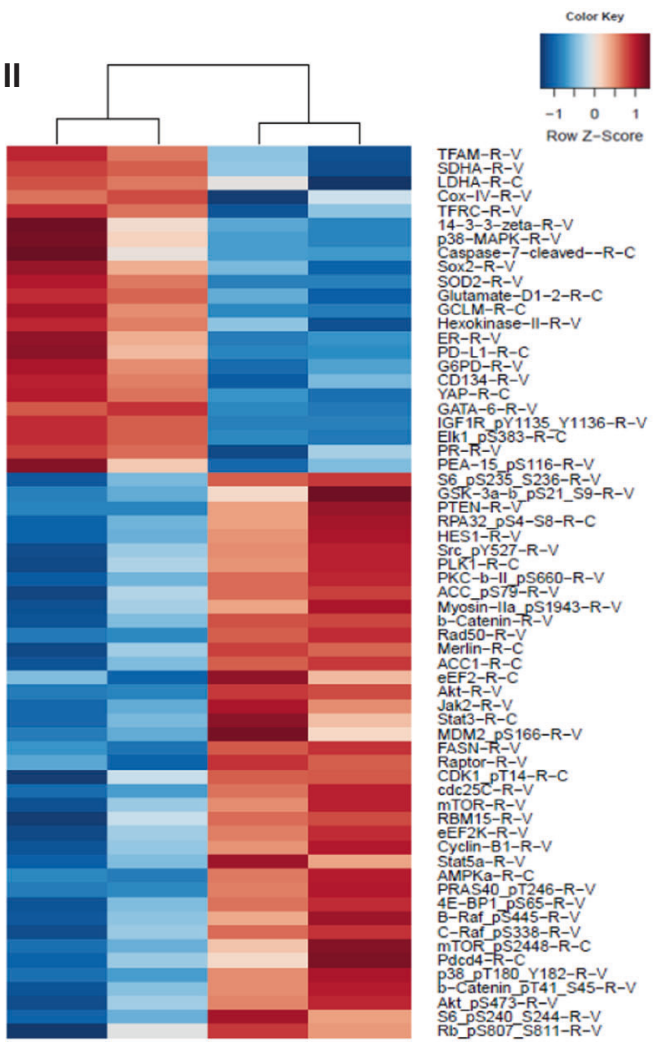

b

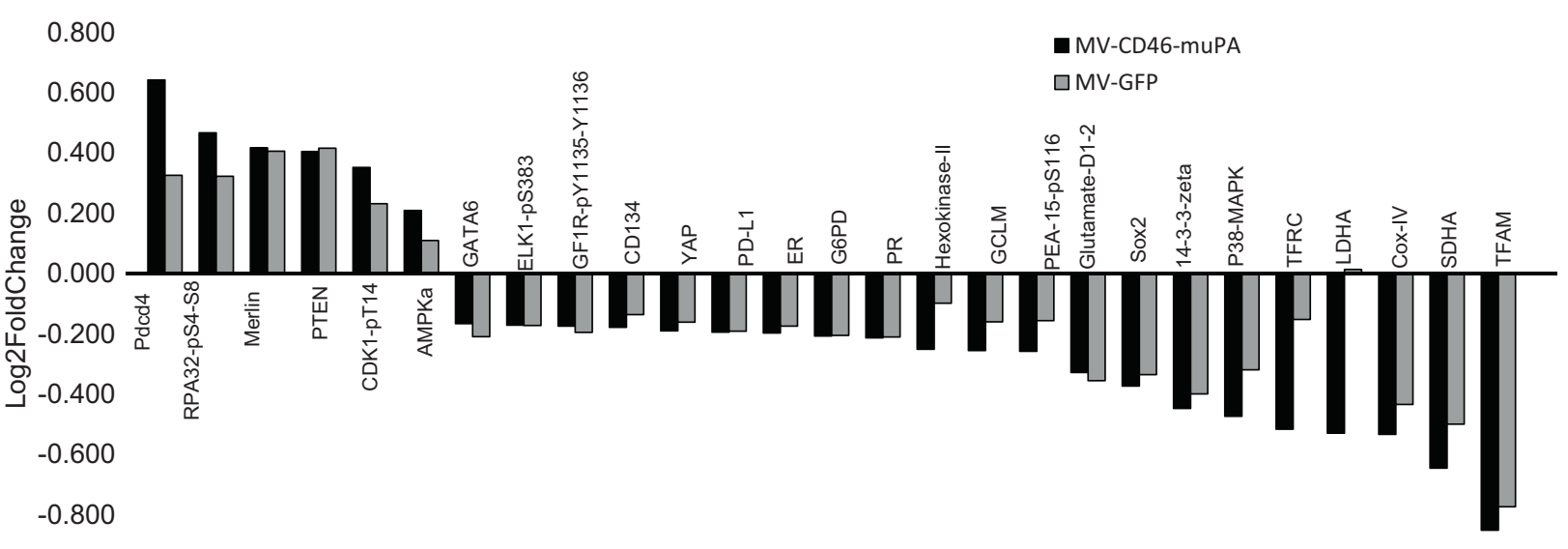

Fig. 6 In vivo effects of MV viral vectors on expression of cancer cell protein pathways. HT-29 tumor-bearing mice were treated with vehicle, MV-GFP or MV-CD46-muPA as in methods section. At day 10 after MV treatment, tumors were resected and frozen and tumor lysates were obtained for RPPA analysis. a Heat map of differentially expressed human proteins involved in cell cycle, proliferation, cell death, and survival pathways between vehicle controls vs. MV-GFP (a. I), and controls vs. MV-CD46-muPA. b Selected up- and downregulated proteins associated with MV-GFP and MV-CD46-muPA treatment. Bars represent average of two independent samples per condition. 
involved in tumor metabolism, a decrease in p38-MAPK, 14-3-3 zeta, PEA-15 p S116, as well as an increase in AMPK, programmed cell death 4 (Pdcd 4), p53 binding protein 1 (53-BP1), and CDK1-pT14 (inhibitory phosphorylation). A decrease in the transcriptional co activator TAZ was observed, which correlates with changes in Merlin and Yap expression, as well as a decrease in PKM-2 (Fig. 6b).

\section{Discussion}

The tumor stroma is a well-recognized barrier to the efficient delivery of antitumor agents, including OVs $[10,35,36]$, and overcoming this limitation is an important, unmet need. In this report, we demonstrate that stromal and tumor targeting by a systemically administered oncolytic MV (MV-CD46-muPA) is associated with improved in vivo antitumor effects compared with MV vectors targeting tumor cells via CD46, and describe the potential mechanisms behind these effects.

MV-CD46-muPA successfully infects, replicates, and induces cytotoxicity in both human (via CD46) and murine cells (via murine uPAR) in a manner similar to species specific viral vectors. MV-CD46-muPA infection was successfully transferred via heterofusion from murine stromal cells (fibroblasts) to human cancer cells (and vice versa) in co-culture models of tumor-stromal interactions, effects associated with tumor cell cytotoxicity, and not observed with single targeted viruses (Fig. 3). These data suggest that stromal cells infected by MV-CD46-muPA can transmit infection to tumor cells, potentially facilitating virus tumor delivery and subsequent tumor cell oncolysis.

The above findings were validated in vivo, where treatment with dual targeted viruses induced enhanced antitumor effects, compared with single targeted oncolytic MVs or vehicle-treated controls, in colon cancer xenograft models (Fig. 4). We have previously reported the in vivo effects of stromal targeted only (MV-muPA) MV vectors compared with tumor only targeted vectors in breast cancer models, showing measurable, but modest effects of stromal only in vivo targeting [27]. The improved in vivo effects were associated with increased tumor viral protein (MV-N) expression, as well as increased tumor cell apoptosis. Evidence of antistromal effects by MV-CD46-muPA in vivo was demonstrated by flow cytometric decrease in murine uPAR-expressing stromal cells, tumor endothelial cells and fibroblasts. The above findings translated into improved outcomes in MV-CD46-muPA-treated mice, compared with the control groups. As the main difference between MVCD46-muPA and MV-GFP is mouse uPAR-mediated murine tissue targeting, the differences in antitumor efficacy can be attributed to the stromal targeting abilities of MV-CD46-muPA.

The possible mechanisms of MV-CD46-muPA improved in vivo oncolytic effects are multiple. First, MV-CD46-muPA targeting of stromal cell components may facilitate virus delivery to tumor cells (Fig. 4c I), leading to increased viral penetration and oncolysis. Second, our results suggest that systemically administered MV-CD46-muPA has direct antistromal effects (Fig. 5). Although both MV-GFP and MVCD46-muPA induced inhibition of tumor cell proliferation and enhanced in vivo apoptosis, the magnitude of the proapoptotic effect was significantly higher in tumors treated by the dual targeted virus. The antistromal effects by MV-CD46muPA further validate a previous report by our group, showing that single targeted, stromal specific MV-muPA (selectively targeting mouse uPAR) induces significant modulation of murine stromal pathways, such as angiogenesis, inflammatory networks, as well as indirect modulation of human cancer cell pathways [27]. In this report, we further characterized the in vivo MV oncolytic mechanisms by functional RPPA of tumors treated by the tumor vs. tumor and stromal targeted MV vectors. We found changes in protein expression induced by both viral vectors, including an increase in PTEN [37], and Merlin [38], known tumor suppressors, and a decrease in transcription factors critical for cell cycle progression and survival, such as GATA-6 [39, 40], ELK-1 [41], YAP [42]. In addition, a significant decrease in mitochondrial transcription factor (TFAM), found to be protumorigenic [43, 44], was also observed. The improved tumor suppressive and pro-apoptotic effects of MV-CD46-mUPA were associated with changes in proteins involved in tumor metabolism, such as AMPK (upregulation), and downregulation of transferrin receptor-1 (TFRC), LDH-A, G6PD, hexokinase II, as well as 14-3-3 zeta downregulation, involved in proliferation, survival and adhesion in multiple cancers [45]. The above data suggest that in addition to improving tumor entry and deposition, stromal targeting by an oncolytic MV modulates stromal-tumor interactions, leading to disruption of tumor cell metabolic and survival pathways. The above (RPPA) results are hypothesis generating, and require further validation in additional in vivo and in vitro models of virus-tumor-stromal interactions, a focus of our current efforts.

The implications of the above findings are multiple and significant. First, our results clearly prove the role of stromal MV targeting in the improved viral delivery into tumors. Second, they emphasize the importance of the tumor microenvironment on tumor progression, and validate the tumor stroma as a target for oncolytic virotherapies in general, and oncolytic MVs in particular. Prior studies have characterized fully retargeted MVs directed against multiple receptors, such as the epithelial receptors Her-2/ 
neu and EpCAM [46], or against CD46 and CD133 [47], among other retargeted oncolytic MVs. Although these strategies enhance and potentiate tumor targeting, they do not address the important question of stromal targeting by MV vectors. MV-CD46-muPA proves the concept that oncolytic MVs can effectively target tumor and stromal cells, leading to enhanced in vivo antitumor efficacy. The current report focuses on the non-immune stroma, including endothelial cells and fibroblasts. There are, however, many other stromal components that can be exploited by OV retargeting strategies, including cells of the immune microenvironment, which is the focus of current and future studies by our group and others.

As unmodified oncolytic MV vectors currently used in the clinic, in human cancer trials, bind tumors via CD46, present in all nucleated cells, we speculate that clinical responses to locally or systemically administered MV vectors $[6,48,49]$ may be explained by MV binding to both tumor and stromal cells. However, although there is published evidence of CD46 overexpression in tumor cells, there is no clear indication that tumor-stromal cells overexpress CD46 compared with non-tumor stroma. As uPAR is biologically important target in tumor and is overexpressed in tumor-stromal cells, in addition to tumor cells $[19,50-52]$, we speculate that human uPAR-directed MV virotherapies may be better suited to overcome the stromal barrier, improve viral penetration, which may lead to improved antitumor effects in human cancers. Therefore, our results provide a scientific rationale for the clinical development of new-generation oncolytic MVs-targeting tumor stroma in addition to tumor cells only, either human uPAR, CD46-huPAR dual targeted MV vectors, or combination of unmodified MV vectors (targeting CD46) and human uPAR.

In conclusion, this report demonstrates for the first time the advantages of stromal targeting by an oncolytic MV for therapeutic gain, including improved viral tumor penetration, direct antistromal effects, and enhanced apoptosis, leading to enhanced in vivo antitumor effects. The above results warrant further investigation and development of dual stromal and tumor targeted OVs for the treatment of human cancers.

Acknowledgements This work was supported by a research grant from the National Cancer Institute (1R01CA149659-01 to J.R.M.), and by the Sylvester Comprehensive Cancer Center (J.R.M.). We acknowledge Oliver Umland, PhD. from the University of Miami/DRI flow cytometry core facility for technical assistance in flow cytometry experiments.

Author contributions Y.J. contributed with the execution of the in vitro and in vivo experiments described in the report. V.C. contributed with the experiments related to RPPA analysis. N.K. contributed with experiments related to flow cytometry quantification of tumor-stromal cells. Y.B. and X.C. contributed with the pathway analysis and preparation of pathway heat maps of RPPA. A.P.E. contributed with the statistical analysis of the in vitro and in vivo experiments. J.R.M. contributed with the design of the viral vector, overall plan, and design of the in vitro and in vivo experiments, analysis of the data and preparation of the manuscript.

\section{Compliance with ethical standards}

Conflict of interest The authors declare that they have no conflict of interest.

Publisher's note Springer Nature remains neutral with regard to jurisdictional claims in published maps and institutional affiliations.

\section{References}

1. Kaufman HL, Kohlhapp FJ, Zloza A. Oncolytic viruses: a new class of immunotherapy drugs. Nat Rev Drug Discov. 2015;14:642-62.

2. Fountzilas C, Patel S, Mahalingam D. Review: Oncolytic virotherapy, updates and future directions. Oncotarget. 2017;8:102617-39.

3. Jhawar SR, Thandoni A, Bommareddy PK, Hassan S, Kohlhapp FJ, Goyal S, et al. Oncolytic viruses-natural and genetically engineered cancer immunotherapies. Front Oncol. 2017;7:202.

4. Patel MR, Kratzke RA. Oncolytic virus therapy for cancer: the first wave of translational clinical trials. Transl Res. 2013;161:355-64.

5. Robinson S, Galanis E. Potential and clinical translation of oncolytic measles viruses. Expert Opin Biol Ther. 2017;17:353-63.

6. Russell SJ, Federspiel MJ, Peng KW, Tong C, Dingli D, Morice WG, et al. Remission of disseminated cancer after systemic oncolytic virotherapy. Mayo Clin Proc. 2014;89:926-33.

7. Aref S, Bailey K, Fielding A. Measles to the rescue: a review of oncolytic measles virus. Viruses. 2016;22:88 . pii: E294.

8. Vaha-Koskela M, Hinkkanen A. Tumor restrictions to oncolytic virus. Biomedicines. 2014;2:163-94.

9. Miller AC, Russell SJ. Heterogeneous delivery is a barrier to the translational advancement of oncolytic virotherapy for treating solid tumors. Virus Adapt Treat. 2014;6:11-31.

10. Wojton J, Kaur B. Impact of tumor microenvironment on oncolytic viral therapy. Cytokine Growth Factor Rev. 2010;21:127-34.

11. Ilkow CS, Marguerie M, Batenchuk C, Mayer J, Ben Neriah D, Cousineau S. et al. Reciprocal cellular cross-talk within the tumor microenvironment promotes oncolytic virus activity. Nat Med. 2015;21:530-6.

12. Peng KW, Myers R, Greenslade A, Mader E, Greiner S, Federspiel MJ, et al. Using clinically approved cyclophosphamide regimens to control the humoral immune response to oncolytic viruses. Gene Ther. 2013;20:255-61.

13. Mader EK, Maeyama Y, Lin Y, Butler GW, Russell HM, Galanis E, et al. Mesenchymal stem cell carriers protect oncolytic measles viruses from antibody neutralization in an orthotopic ovarian cancer therapy model. Clin Cancer Res. 2009;15:7246-55.

14. Mok W, Boucher Y, Jain RK. Matrix metalloproteinases-1 and -8 improve the distribution and efficacy of an oncolytic virus. Cancer Res. 2007;67:10664-8.

15. Nakamura T, Peng KW, Harvey M, Greiner S, Lorimer IA, James $\mathrm{CD}$, et al. Rescue and propagation of fully retargeted oncolytic measles viruses. Nat Biotechnol. 2005;23:209-14.

16. Toro Bejarano MMJ. Targeting tumor vasculature through oncolytic virotherapy: recent advances. Oncol Virother. 2015;4 (November): 169-81, 112015.

17. Jing Y, Tong C, Zhang J, Nakamura T, Iankov I, Russell SJ, et al. Tumor and vascular targeting of a novel oncolytic measles virus 
retargeted against the urokinase receptor. Cancer Res. 2009;69:1459-68.

18. Jing Y, Zaias J, Duncan R, Russell SJ, Merchan JR. In vivo safety, biodistribution and antitumor effects of UPAR retargeted oncolytic measles virus in syngeneic cancer models. Gene Ther. 2014;21:289-97.

19. Akahane T, Ishii M, Ohtani H, Nagura H, Toyota T. Stromal expression of urokinase-type plasminogen activator receptor (uPAR) is associated with invasive growth in primary liver cancer. Liver. 1998;18:414-19.

20. Bianchi E, Cohen RL, Thor AT, Todd RF,3rd, Mizukami IF, Lawrence DA, et al. The urokinase receptor is expressed in invasive breast cancer but not in normal breast tissue. Cancer Res. 1994;54:861-66.

21. Hildenbrand R, Schaaf A. The urokinase-system in tumor tissue stroma of the breast and breast cancer cell invasion. Int J Oncol. 2009;34:15-23.

22. Hofmann R, Lehmer A, Buresch M, Hartung R, Ulm K. Clinical relevance of urokinase plasminogen activator, its receptor, and its inhibitor in patients with renal cell carcinoma. Cancer. 1996;78:487-92.

23. Kroon ME, Koolwijk P, van der Vecht B, van Hinsbergh VW. Urokinase receptor expression on human microvascular endothelial cells is increased by hypoxia: implications for capillary-like tube formation in a fibrin matrix. Blood. 2000;96:2775-83.

24. Romer J, Nielsen BS, Ploug M. The urokinase receptor as a potential target in cancer therapy. Curr Pharm Des. 2004;10:2359-76.

25. Xu Y, Hagege J, Doublet JD, Callard P, Sraer JD, Ronne E, et al. Endothelial and macrophage upregulation of urokinase receptor expression in human renal cell carcinoma. Hum Pathol. 1997;28:206-13.

26. Jing Y, Bejarano MT, Zaias J, Merchan JR. In vivo anti-metastatic effects of uPAR retargeted measles virus in syngeneic and xenograft models of mammary cancer. Breast Cancer Res Treat. 2015;149:99-108.

27. Jing Y, Chavez V, Ban Y, Acquavella N, El-Ashry D, Pronin A, et al. Molecular effects of stromal-selective targeting by uPARretargeted oncolytic virus in breast cancer. Mol Cancer Res. 2017;15:1410-20.

28. Merchan JR, Tang J, Hu G, Lin Y, Mutter W, Tong C, et al. Protease activity of urokinase and tumor progression in a syngeneic mammary cancer model. J Natl Cancer Inst. 2006;98:756-64.

29. Parks CL, Lerch RA, Walpita P, Sidhu MS, Udem SA. Enhanced measles virus cDNA rescue and gene expression after heat shock. J Virol. 1999;73:3560-6.

30. Drews-Elger K, Brinkman JA, Miller P, Shah SH, Harrell JC, da Silva TG, et al. Primary breast tumor-derived cellular models: characterization of tumorigenic, metastatic, and cancer-associated fibroblasts in dissociated tumor (DT) cultures. Breast Cancer Res Treat. 2014;144:503-17

31. Smyth GK. Linear models and empirical bayes methods for assessing differential expression in microarray experiments. Stat Appl Genet Mol Biol. 2004;3:Article3.

32. Benjamini Y, Hochberg Y. Controlling the false discovery rate - a practical and powerful approach to multiple testing. J Roy Stat Soc B Met. 1995;57:289-300.

33. Castleton A, Dey A, Beaton B, Patel B, Aucher A, Davis DM, et al. Human mesenchymal stromal cells deliver systemic oncolytic measles virus to treat acute lymphoblastic leukemia in the presence of humoral immunity. Blood. 2014;123:1327-35.

34. Pallangyo CK, Ziegler PK, Greten FR. IKKbeta acts as a tumor suppressor in cancer-associated fibroblasts during intestinal tumorigenesis. J Exp Med. 2015;212:2253-66.
35. Jain RK. Vascular and interstitial barriers to delivery of therapeutic agents in tumors. Cancer Metastasis Rev. 1990;9:253-66.

36. Tredan O, Galmarini CM, Patel K, Tannock IF. Drug resistance and the solid tumor microenvironment. J Natl Cancer Inst. 2007;99:1441-54.

37. Milella M, Falcone I, Conciatori F, Cesta Incani U, Del Curatolo A, Inzerilli N, et al. PTEN: multiple eunctions in human malignant tumors. Front Oncol. 2015;5:24.

38. Cooper J, Giancotti FG. Molecular insights into NF2/Merlin tumor suppressor function. FEBS Lett. 2014;588:2743-52.

39. Belaguli NS, Aftab M, Rigi M, Zhang M, Albo D, Berger DH. GATA6 promotes colon cancer cell invasion by regulating urokinase plasminogen activator gene expression. Neoplasia. 2010;12:856-65.

40. Lin L, Bass AJ, Lockwood WW, Wang Z, Silvers AL, Thomas DG, et al. Activation of GATA binding protein 6 (GATA6) sustains oncogenic lineage-survival in esophageal adenocarcinoma. Proc Natl Acad Sci USA. 2012;109:4251-6.

41. Rao VN, Reddy ES. elk-1 proteins interact with MAP kinases. Oncogene. 1994;9:1855-60.

42. Ehmer U, Sage J. Control of proliferation and cancer growth by the hippo signaling pathway. Mol Cancer Res. 2016;14:127-40.

43. Araujo LF, Siena ADD, Placa JR, Brotto DB, Barros II, Muys BR, et al. Mitochondrial transcription factor A (TFAM) shapes metabolic and invasion gene signatures in melanoma. Sci Rep. 2018;8:14190.

44. Xie D, Wu X, Lan L, Shangguan F, Lin X, Chen F, et al. Downregulation of TFAM inhibits the tumorigenesis of non-small cell lung cancer by activating ROS-mediated JNK/p38MAPK signaling and reducing cellular bioenergetics. Oncotarget. 2016;7:11609-24.

45. Matta A, Siu KW, Ralhan R. 14-3-3 zeta as novel molecular target for cancer therapy. Expert Opin Ther Targets. 2012;16:515-23.

46. Hanauer JR, Gottschlich L, Riehl D, Rusch T, Koch V, Friedrich $\mathrm{K}$, et al. Enhanced lysis by bispecific oncolytic measles viruses simultaneously using HER2/neu or EpCAM as target receptors. Mol Ther Oncolytics. 2016:3:16003.

47. Kleinlutzum D, Hanauer JDS, Muik A, Hanschmann KM, Kays SK, Ayala-Breton C, et al. Enhancing the oncolytic activity of CD133-targeted measles virus: receptor extension or chimerism with vesicular stomatitis virus are most effective. Front Oncol 2017;7:127.

48. Galanis E, Hartmann LC, Cliby WA, Long HJ, Peethambaram PP, Barrette BA, et al. Phase I trial of intraperitoneal administration of an oncolytic measles virus strain engineered to express carcinoembryonic antigen for recurrent ovarian cancer. Cancer Res. 2010;70:875-82.

49. Heinzerling L, Kunzi V, Oberholzer PA, Kundig T, Naim H, Dummer R. Oncolytic measles virus in cutaneous T-cell lymphomas mounts antitumor immune responses in vivo and targets interferon-resistant tumor cells. Blood. 2005;106:2287-94.

50. Boonstra MC, Verbeek FP, Mazar AP, Prevoo HA, Kuppen PJ, van de Velde CJ, et al. Expression of UPAR in tumor-associated stromal cells is associated with colorectal cancer patient prognosis: a TMA study. BMC Cancer. 2014;14:269.

51. Dublin E, Hanby A, Patel NK, Liebman R, Barnes D. Immunohistochemical expression of UPA, uPAR, and PAI-1 in breast carcinoma. Fibroblastic expression has strong associations with tumor pathology. Am J Pathol. 2000;157:1219-27.

52. Hildenbrand R, Schaaf A, Dorn-Beineke A, Allgayer H, Sutterlin $\mathrm{M}$, Marx A, et al. Tumor stroma is the predominant uPA-, uPAR-, PAI-1-expressing tissue in human breast cancer: prognostic impact. Histol Histopathol. 2009;24:869-77. 